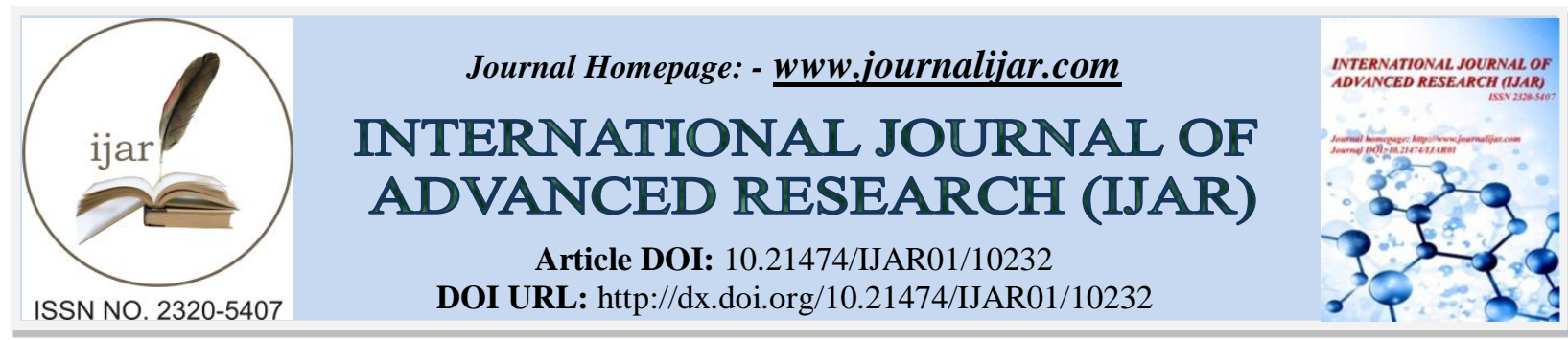

RESEARCH ARTICLE

\title{
LE RHABDOMYOSARCOME EMBRYONNAIRE DU COL UTERIN ET LA TUMEUR DE GRANULOSA ADULTE : UNE ASSOCIATION TRES RARE A PROPOS D'UN CAS ET REVUE DE LA LITTÉRATURE
}

\author{
K. Kriouile, H. Afalah, S. Jayi, F.Z Fdili Alaoui, H. Chaara and My A. Melhouf
}

Service De Gynécologie Obstétrique II, CHU Hassan II, Fès. Maroc.

\section{Manuscript Info}

\section{Manuscript History}

Received: 12 October 2019

Final Accepted: 14 November 2019

Published: December 2019

Key words:-

Rhabdomyosarcome, Tumeur De La Granulosa, Col Utérin, Jeune Fille

\begin{abstract}
Le rhabdomyosarcome (RMS) du col utérin fait partie des types histologiques rares des cancers du col, qu'on observe généralement chez la jeune fille ou la femme en période d'activité génitale. La stratégie thérapeutique se base sur l'association des trois modalités thérapeutiques (chimiothérapie-radiothérapie-chirurgie) vu l'agressivité de la maladie. Les tumeurs de la granulosa sont des tumeurs rares de l'ovaire, appartenant au groupe des tumeurs du stroma et cordons sexuels. Elles se développent à partir des cellules granulomatoses et thécales. Elles sont subdivisées en tumeurs adultes (95\%) et juvéniles. Le plus souvent, elles sont oestrogénosécrétantes, à l'origine de signes cliniques variables en fonction de l'âge : puberté précoce, irrégularité menstruelle et métrorragies. L'association de ces deux tumeurs est exceptionnelle. Nous rapportons ainsi un cas d'une jeune fille de 18 ans admise pour métrorragies abondantes avec à l'examen la présence d'une masse polypoide extériorisée par la vulve et dont la biopsie est revenue en faveur d'un rhabdomyosarcome du col, l'exploration radiologique a objectivé une tumeur ovarienne associée, dont le résultat anatomopathologique après extirpation est revenu en faveur d'une tumeur de la granulosa adulte. A travers ce fait clinique nous allons décrire les caractéristiques anatomo-cliniques de ces tumeurs et étudier les différents facteurs pronostic afin de déterminer une attitude thérapeutique correcte. Le RMS du col utérin est une tumeur rare qui survit essentiellement chez la jeune fille. L'extension est surtout locorégionale. Le traitement consiste en un geste chirurgical allant d'un éventuel traitement conservateur jusqu'au traitement radical associé à une chimiothérapie péri-opératoire. La place de la radiothérapie demeure imprécise.

Copy Right, IJAR, 2019,. All rights reserved.
\end{abstract}

\section{Introduction:-}

Le rhabdomyosarcome botryoïde du col utérin est une tumeur rare de la femme en activité génitale. Une centaine de cas ont été publiés dans cette localisation. Elle survient électivement chez la femme jeune. Il se manifeste surtout par des hémorragies vaginales.L'aspect macroscopique est celui d'une tumeur polypoïde appendue au col utérin réalisant le classique aspect en «grappes de raisins»[1]. Si le traitement autrefois était basé sur une chirurgie agressif allant jusqu'à l'exentération pelvienne, on préconise actuellement un traitement conservateur parfois limité à une conisation, associé à la chimiothérapie et dont les résultats sont encourageants.

\section{Corresponding Author:- K. Kriouile}

Address:- Service De Gynécologie Obstétrique II, CHU Hassan II, Fès. Maroc. 
Nous rapportons un cas d'une jeune patiente de 18 ans admise pour prise en charge d'une tumeur polypoïde appendue au col utérin. Nous insistons à travers cette observation sur les progrès diagnostiques et thérapeutiques facilitant la prise en charge de cette tumeur rare du col utérin.

\section{Observation:-}

Patiente de 18 ans, de petite taille $1,40 \mathrm{~m}$. Admise pour métrorragies de faible à moyenne abondance évoluant depuis un an. L'examen clinique trouve une patiente stable sur le plan hémodynamique avec la présence d'une masse d'aspect polypoide extériorisée par la vulve (Figure1).

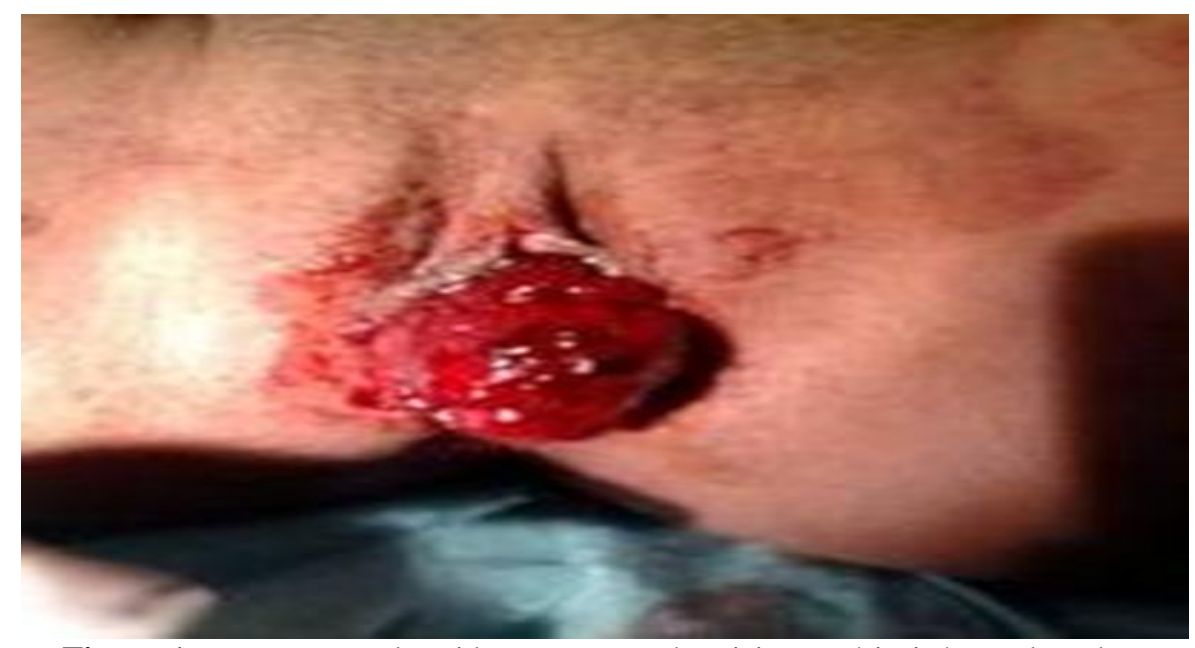

Figure 1:- La masse polypoide en grappes de raisins extériorisée par la vulve.

\section{A l'échographie pelvienne:}

on a objectivé la présence d'une image échogène avec des zones hypo-échogènes comblant tout le vagin, vascularisée faisant évoquée un gros polype, avec un ovaire droit augmenté de taille.

\section{L'IRM pelvienne:}

a objectivé une volumineuse masse endovaginale correspondant probablement à un polype accouché par le col mesurant 50/47/23 mm, associé à un épaississement endométrial de $9 \mathrm{~mm}$, avec la présence d'une image hétérogène de 31/34/27 mm ovarienne droite évoquant en premier une tumeur de la granulosa juvénile vu l'association au terrain d'hyperoestrogénie.

On a réalisé une biopsie de la masse prolabée par la vulve:

revenant en faveur d'un aspect évoquant un rhabdomyosarcome embryonnaire de type botryoïde (Figure 2).

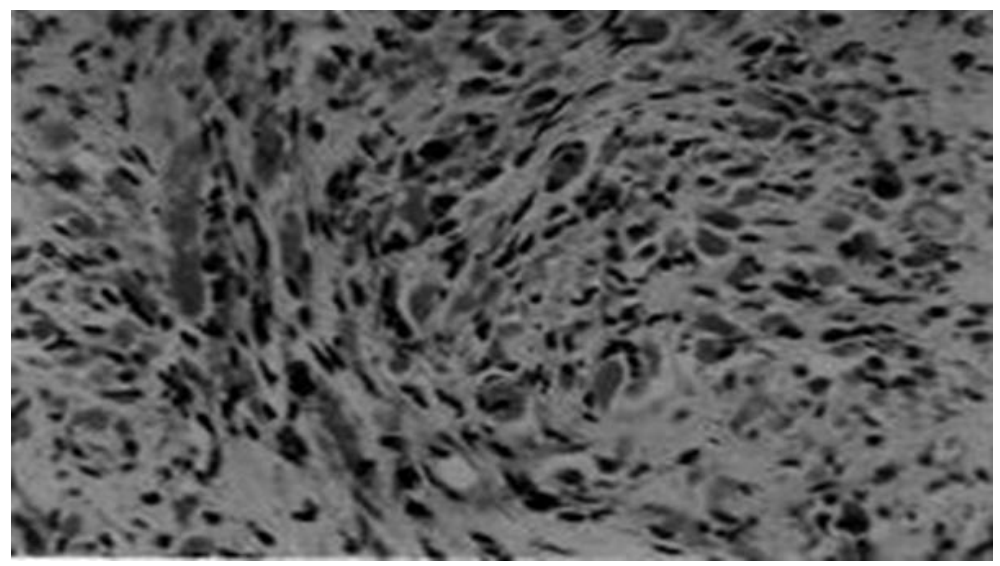

Figure 2:- Aspect histologique objectivant un tissu conjonctif siège de cellules indifférenciées étoilées à noyau hyperchromatique. 


\section{La Tdm Tap:}

a objectivé une masse tissulaire de l'ovaire droit mesurant $40 \mathrm{~mm}$ de diamètre rehaussée de façon hétérogène faisant évoquer une métastase ovarienne. Avec la présence d'un volumineux goitre hétérogène multinodulaire légèrement plongeant pouvant être une localisation secondaire (Figure 3).

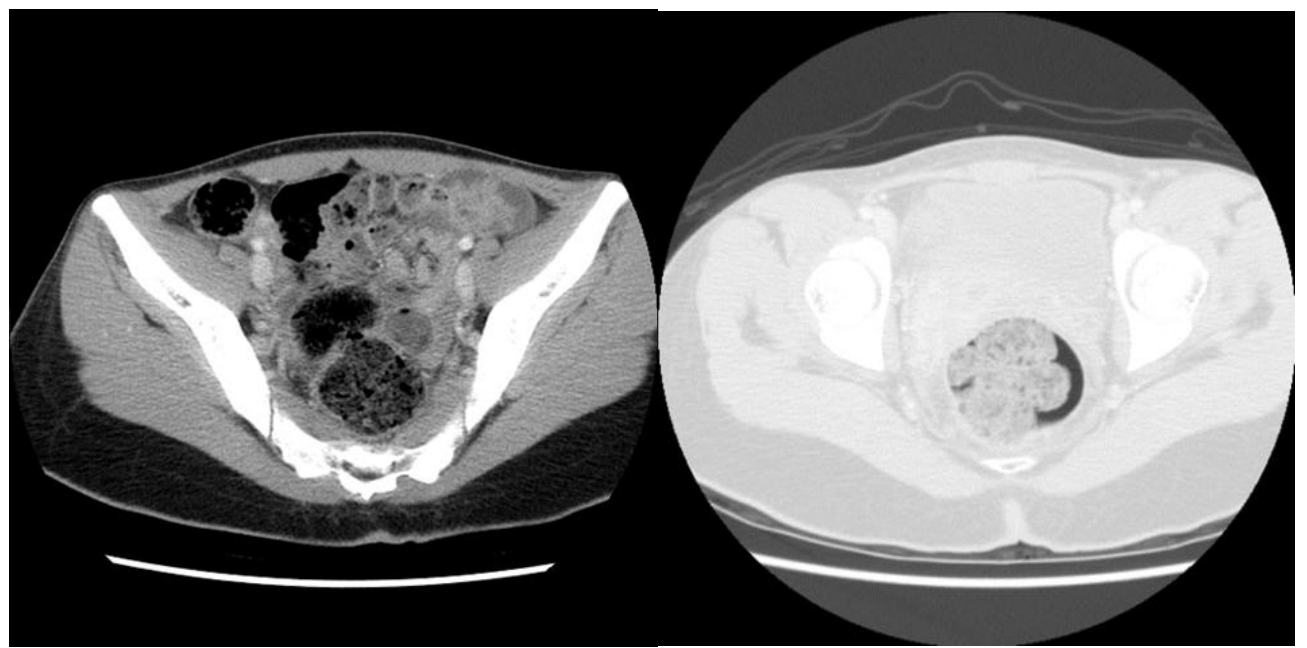

Figure 3:- Coupes transversales de la TDM abdomino-pelvienne objectivant la masse tumorale dans la région cervicale.

\section{L'échographie cervicale:}

est revenu en faveur d'un aspect échographique d'un goitre multi nodulaire dont la majorité des lésions sont kystiques, situées à gauche. Le bilan thyroïdien : est revenu normal.

Le dossier a été ensuite discuté en Réunion de Concertation Multidisciplinaire (RCP), la décision du staff RCP était de : faire une cytoponction du nodule thyroidien, dont l'étude anatomopathologique est revenue en faveur de lésion bénigne.

La patiente a bénéficié d'une laparotomie exploratrice objectivant : un ovaire droit à parois épaissie et induré, ovaire gauche d'aspect normal. On a réalisé une trachelectomie associée à une ovariectomie droite avec extemporanée et des biopsies multiples. La patiente a bénéficié aussi d'une isthmo-lobectomie gauche par les ORL.

\section{Resultats Anapath:-}

Piece De De Trachelectomie:

Aspect histologique d'un rhabdomyosarcome embryonnaire de type botryoïde. Les limites de résection sont saines.

\section{Masse Ovarienne Droite:}

Tumeur de la granulosa adulte associée à une hyperplasie de cellules de Leidig.

\section{Biopsie De L'ovaire Gauche:}

Parenchyme ovarien sensiblement normal.

\section{Biopsie Epiplö̈que Et Goutiere Parieto-Colique Droite Et Gauche:}

Tissu fibro-adipeux sensiblement normal

\section{Piece De La Thyroide:}

Hyperplasie bénigne. Le dossier a été rediscuté en RCP la décision était de faire une chimiothérapie néoadjuvante puis de compléter le geste chirurgical par une salpingectomie avec omentectomie infracolique pour compléter le staging chirurgical de la tumeur de granulosa.

La patiente a bénéficié d'une hystéroscopie diagnostic première afin d'innocenté l'endomètre qui est revenu sans particularité, complétée d'une salpingectomie droite avec omentectomie infracolique. 


\section{Résultat Anatomopathologie:- \\ Pièce de salpingectomie droite:}

granulome à corps étranger tubaire, absence de malignité.

\section{Pièce d'omentectomie:}

tissu graisseux sensiblement normal La patiente est adressée par la suite en oncologie, ayant bénéficiée d'une chimiothérapie adjuvante type VAC pendant 1 an, puis mise sous surveillance pendant 1 an. La patiente a présenté une rechute métastatique au niveau péritonéal, mise sous polychimiothérapie palliative type VAI. La patiente est décédée 3 mois plus tard par défaillance multi-vicérale.

\section{Discussion:-}

On décrit sous le nom de rhabdomyosarcome (RMS) des tumeurs mésenchymateuses malignes de degré de différenciation variable, et d'origine mésodermique. Ces tumeurs se développent électivement au niveau du tractus urogénital, souvent au niveau du vagin et de la vulve. La localisation cervicale est rarissime.

L'âge moyen de diagnostic se situe entre 10 et 20 ans[2]. Il touche la jeune fille et la femme en activité génitale.

Les circonstances habituelles du diagnostic sont dominées par des métrorragies récidivantes. Au stade initial, les lésions peuvent être asymptomatiques ou prendre un aspect de polype bénin. Ce qui explique l'absence habituelle de diagnostic précoce.

Souvent, il s'agit d'un polype muqueux récidivant très rapidement après son ablation. L'examen gynécologique retrouve une tuméfaction de taille variable appendue au col utérin, d'aspect polypoïde réalisant le classique aspect en « grappe de raisins» [3]. C'est le cas chez notre jeune patiente.

Sur le plan histopathologique, différents types histologiques sont définis actuellement après confrontation des classifications antérieurement proposées par l'intergroup rhabdomyosarcoma study (IRS), la société internationale d'oncologie pédiatrique (SIOP), le national cancer institue (NCI) : Le rhabdomyosarcome embryonnaire, anaplasique et alvéolaire [2-4]. Le groupe embryonnaire comporte deux sous-groupes: le rhabdomyosarcome botryoïde et à cellule fusiforme [5].

Le rhabdomyosarcome botryoïde est une forme du rhabdomyosarcome embryonnaire qui se caractérise par la présence d'une bande cambiale sous-épithéliale et par une différenciation musculaire striée représentée par une double striation intra-cytoplasmique. L'immunomarquage confirme l'origine musculaire de la tumeur par l'expression de la desmine et de l'actine [5,6], par l'étude ultrastructurale et au besoin par la mise en évidence de myoglobine à l'immunohistochimie.

Le RMS du col utérin est une tumeur à extension locorégionale massive, l'évaluation de l'extension de cette tumeur peut se faire selon la classification de l'IRSG [7]. Les métastases sont rarement observées. Par contre les récidives sont constantes après exérèse limitée, comme le cas de notre patiente.

Le traitement du rhabdomyosarcome botryoïde du col utérin n'est pas codifié. Jusqu'aux années 1970, l'exentération pelvienne était le traitement chirurgical standard. Actuellement, ce type de chirurgie est de moins en moins utilisé, la chirurgie conservatrice étant la tendance actuelle. Pour Zeisler et al., chez les patientes du groupe 1 (Tableau 1), qui n'ont pas de résidus microscopique après chirurgie et des facteurs de pronostic favorable, tels qu'un polype unique, le type embryonnaire et l'absence d'envahissement du myomètre, une chirurgie conservatrice ou une simple hystérectomie peut être suffisante. Pour certains auteurs, le bénéfice d'une chimiothérapie adjuvante n'est pas encore démontré, pour d'autres, son efficacité serait indéniable et devrait être préconisée, même en cas de tumeur localisée. Le concept de chimiothérapie adjuvante a été introduit vers les années 1960. Il s'agit d'une triple association : vincristine, dactinomycine et cyclophosphamide (VAC) [8]. 


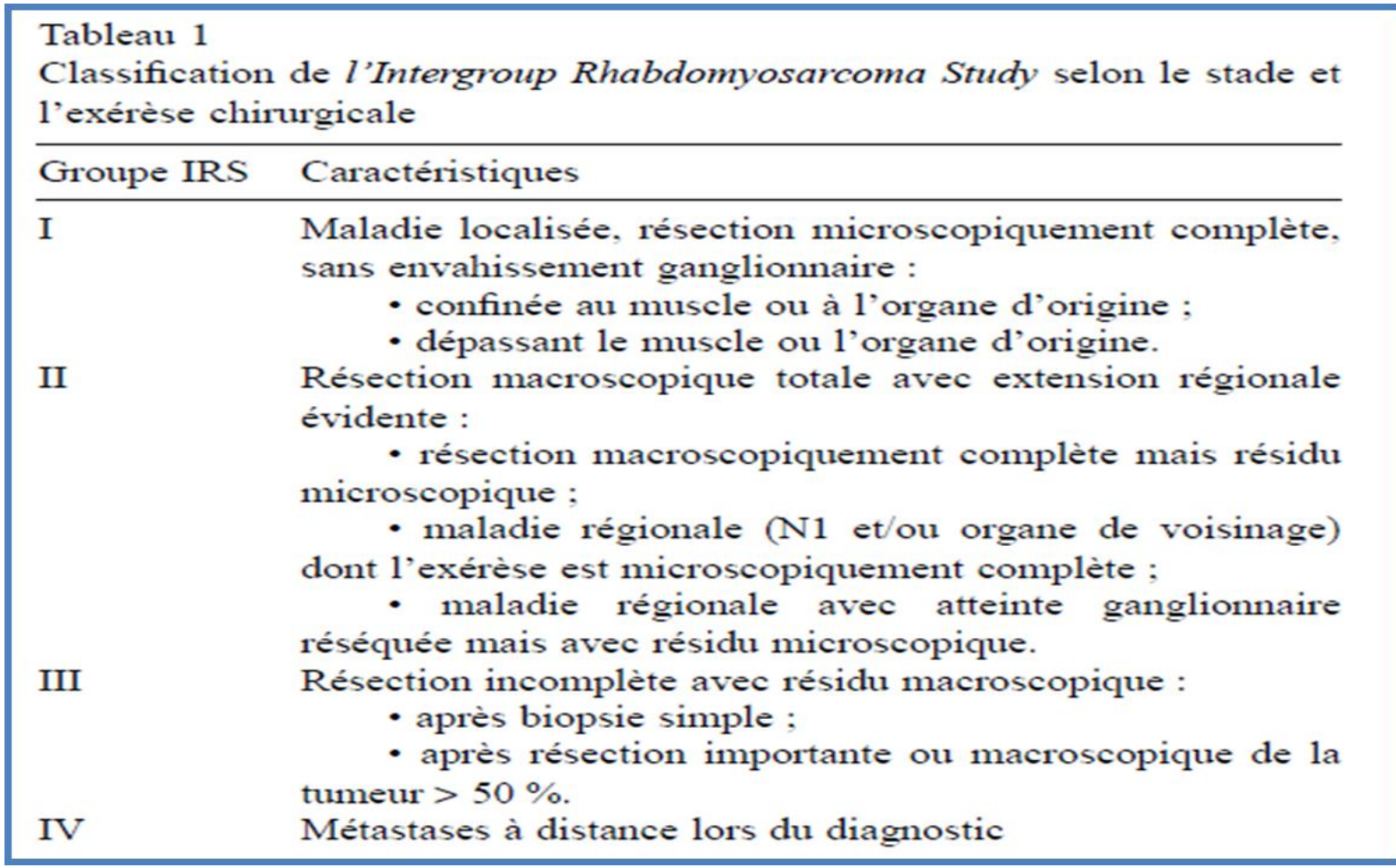

En 1988, Daya et Scully ont revu 21 cas de rhabdomyosarcome botryoïde du col utérin, ils ont rapporté que l'association vincristine et dactinomycine (VA) était aussi efficace que le VAC et que la toxicité du VA était inférieure à celle du VAC. Pour certains auteurs, l'adjonction de doxorubicine au VAC chez les patientes des groupes III et IV, ne modifierait pas les résultats du VAC seul. La place de la radiothérapie postopératoire n'est pas démontrée [9]. La majorité des auteurs s'accordent sur le fait que la radiothérapie n'est pas indiquée chez les patientes du groupe I de l'IRS. La radiothérapie n'a pas été effectuée dans le cas que nous rapportons.

Le pronostic du rhabdomyosarcome de l'adulte n'est pas bien connu du fait de la rareté de cette pathologie à l'âge adulte. Selon Esnola, en matière de rhabdomyosarcome de l'adulte, la présence de métastases au moment du diagnostic et une mauvaise réponse à la chimiothérapie sont deux éléments fortement évocateurs d'un pronostic défavorable [10].

Le facteur pronostic le plus important en cas de rhabdomyosarcomebotryoïde du col, semble être l'extension tumorale au moment du diagnostic. Cette localisation serait de pronostic plus favorable que tout autre rhabdomyosarcome génital et ceci d'autant plus que le polype est unique et que la résection est complète.

\section{Conclusion:-}

Le RMS du col utérin est une tumeur rare qui survient essentiellement chez la jeune fille. L'extension est surtout loco-régionale. Le traitement consiste en un geste chirurgical à minima associé à une chimiothérapie péri opératoire.La place de la radiothérapie demeure imprécise.

\section{Références:-}

1. Semlali.H, Jouhadi.H, Attar.H, Sahraoui.S, Benider.A. A rare cases of rhabdomyosarcoma of the uterine cervix: about a case and review of the literature.Pan African Medical Journal. 2016; 25:166 doi:10.11604/pamj.2016.25.166.8629

2. Saadi I, Errihani H, Haddadi K. Rhabdomyosarcome botryoïde du col utérin: à propos d'un cas. Cancer Radiother. 2002 Dec; 6(6): 363-5.

3. Maingnen C, Hugol D, Caulet S. Rhabdomyosarcomebotryoide du col utérin: étude anatomo-clinique d'un cas. Ann Pathol. 1993; 13(1): 40-4. 
4. Atlante M, Dioniso B, Cioni M. Sarcoma botryoides of the uterine cervix in a young woman: case report. Eur J GynaecolOncol. 2000; 21(5): 504-6.

5. Philippe-Chomette P, Orbach D. Rhabdomyosarcomes du sinus urogénital de l'enfant. Ann Urol (Paris). 2006 Oct; 40(5): 280-96.

6. Matsuura Y, Kashimura M, Hatanaka K. Sarcoma botryoides of the cervix: report of a case with cytopathologic findings. Acta Cytol. 1999 May-Jun; 43(3): 475-80.

7. Maurer HM, Beltangady M, Gehan EA,The Intergroup Rhabdomyosarcoma Study-1. A final report. Cancer 1988; 61:209-20.

8. JiXinQiang, Osamu Takahashi, Junichi Hatazawa, Akihiro Karube, NorikiOhyama, et al. Sarcoma botryoides of the uterine cervix: A case report. J ObstGynaecol Res (Vol 24) n 3 1998:197-201.

9. Little DJ, Ballo MT, Zagars GK, Pisters PW, Patel SR, El-Naggar AK, et al. Adult rhabdomyosarcoma: outcome following multimodality treatment. Cancer Jul 15 2002;95(2):3777-88.

10. Esnola NF, Rubin BP, Baldini EH, Vasudevan N, Demetri GD,Fletcheret CD, et al. Response to chemotherapy and predictors of suvival in adult rhabdomyosarcoma. Ann Surg 2001;234(2):215-23. 